\title{
Video Forgery and Motion Editing
}

\author{
Joseph C. Tsai ${ }^{1}$ and Timothy K. Shih ${ }^{2}$ \\ ${ }^{1}$ Multimedia Information Network Lab \\ Tamkang University, Taiwan \\ kkiceman@gmail.com \\ ${ }^{2}$ Multimedia Information Network Lab \\ National Central University, Taiwan \\ TimothyKShih@gmail.com
}

\begin{abstract}
Video Forgery is a technique for generating fake video by altering, combining, or creating new video contents. We change the behavior of actors in a video. For instance, the outcome of a 100-meter race in the Olympic Game can be falsified. We track objects and segment motions using a modified mean shift mechanism. The resulting video layers can be played in different speeds and at different reference points with respect to the original video. In order to obtain a smooth movement of target objects, a motion interpolation mechanism is proposed based on reference stick figures (i.e., a structure of human skeleton) and video inpainting mechanism. The video inpainting mechanism is performed in a quasi-3D space via guided 3D patch matching. Interpolated target objects and background layers are fused. It is hard to tell whether a falsified video is the original.

In addition, in this talk, we demonstrate a new technique to allow users to change the dynamic texture used in a video background for special effect production. For instance, the dynamic texture of fire, smoke, water, cloud, and others can be edited through a series of automatic algorithms. Motion estimations of global and local textures are used. Video blending techniques are used in conjunction with a color balancing technique. The editing procedure will search for suitable patches in irregular shape blocks, to reproduce a realistic dynamic background, such as large waterfall, fire scene, or smoky background. The technique is suitable for making science fiction movies.

We demonstrate the original and the falsified videos in our website at http://www.csie.ncu.edu.tw/ tshih. Although video falsifying may create a moral problem, our intension is to create special effects in movie industry.
\end{abstract}

Keywords: Video Forgery, Object Tracking, Motion Estimation, Special Effect Production. 